\section{Aspectos demográficos e mortalidade de populações indígenas do Estado do Mato Grosso do Sul, Brasil}

\author{
Demographic characteristics and mortality among \\ indigenous peoples in Mato Grosso do Sul \\ State, Brazil
}

\author{
1 Universidade Estadual de \\ Londrina, Londrina, Brasil. \\ Correspondência \\ M. E. V. Ferreira \\ Universidade Estadual de \\ Londrina. \\ Rua Tamanday 383, apto \\ 403, Santa Maria, $R S$ \\ 97060-540, Brasil. \\ mariaevanirvf@gmail.com
}

\section{Abstract}

The present study aimed to assess mortality rates and related demographic factors among indigenous peoples in the State of Mato Grosso do Sul, Central-West Brazil, compared to the State's general population. Mortality rates were estimated based on data obtained from the Health Care Database for Indigenous Peoples and monthly patient care records as well as demographic data from the Brazilian Unified National Health System (SUS) and mortality data from the SUS Mortality Database. Compared to the overall population, among indigenous peoples there were proportionally more individuals under 15 years of age and fewer elderly, besides higher mortality rates at early ages and from infectious and parasitic diseases. Indigenous men showed significantly higher mortality rates from external causes and respiratory and infectious diseases, while among women the mortality rates from external causes and infectious diseases were higher. Suicide rates among young indigenous individuals were also particularly alarming. Indigenous people's health conditions are worse than those of the general population in Mato Grosso do Sul.

South American Indians; Indigenous Population; Health Status Indicators; Health Inequalities
Maria Evanir Vicente Ferreira 1

Tiemi Matsuo 1

Regina Kazue Tanno de Souza 1

\section{Introdução}

As condições desfavoráveis de saúde das populações indígenas no Brasil são evidenciadas pela desigualdade nas taxas de mortalidade entre esses povos e a população em geral. O perfil de morbidade e mortalidade dos povos indígenas no Brasil, historicamente, era conhecido pela predominância das doenças infecciosas e parasitárias ${ }^{1}$. Atualmente, juntamente com as doenças infecciosas, devido às mudanças intensas em sua relação com a terra, o trabalho, a urbanização e a incorporação de novos hábitos culturais, como o consumo de alimentos industrializados e diminuição das atividades físicas, as doenças crônicas não transmissíveis, tais como a obesidade, hipertensão e diabetes mellitus, tornam-se cada vez mais importantes nessas populações 1,2. Somamse a essas causas os transtornos mentais e comportamentais como o alcoolismo, a violência, os suicídios e os acidentes de trânsito 3 .

Estudos envolvendo a questão da desigualdade desfavorável dos indígenas como tema de saúde coletiva têm aumentado substancialmente nos últimos anos 4,5,6,7. A criação do Subsistema de Atenção à Saúde Indígena, em 1999, por intermédio da Fundação Nacional de Saúde (FUNASA), que assumiu a responsabilidade de estruturar este sistema, articulado ao Sistema Único de Saúde (SUS) 8, tem possibilitado estudos mais abrangentes. Apesar da fragilidade dos registros do subsistema, o cálculo de indicadores 
demográficos e de saúde a partir dos dados disponíveis vem permitindo análises contextuais e macrossociais da situação de saúde indígena.

O Estado do Mato Grosso do Sul, que se localiza no sul da Região Centro-oeste do Brasil, é o segundo estado em número de índios, superado apenas pelo Estado do Amazonas. Segundo a Fundação Nacional de Saúde 9, esses índios pertencem a oito etnias (Guarani, Kaiowá, Terena, Kadiwéu, Kinikinau, Guató, Ofaié e Atikum). A área territorial é equivalente a 590 mil hectares, sendo que $90 \%$ dessas terras pertencem aos índios Kadiwéu 9.

Embora nesse estado se concentre o segundo maior contingente populacional indígena do país, existem poucos estudos publicados recentemente que analisem as condições de saúde desses povos. A maioria dessas publicações tem destacado a história das populações e questões territoriais 10,11, migração 12 , desnutrição infantil 13,14,15,16 e morbidades 17,18,19, envolvendo as etnias Guarani, Terena e Ofaié. Sobre mortalidade foram encontrados alguns estudos nessa população, que se referiam apenas à mortalidade por suicídio nos Kaiowá/Guarani 20,21.

Considerando-se a possibilidade de analisar as condições de saúde, a partir das estatísticas vitais, com a criação de um sistema de informação específico para os indígenas nos moldes do Sistema de Informações sobre de Mortalidade (SIM), a presente investigação objetivou analisar os aspectos demográficos e padrão de mortalidade das populações indígenas residentes nas terras indígenas do Estado do Mato Grosso do Sul comparativamente ao padrão apresentado pela população total do estado, com vistas a contribuir no aprofundamento dos conhecimentos acerca das condições de saúde das populações indígenas.

\section{Metodologia}

O campo de estudo foi a área de abrangência do Distrito Sanitário Especial Indígena (DSEI), cuja sede se situa no Município de Campo Grande. Vinte e sete municípios compõem a área de abrangência desse DSEI. Fazem parte da rede deste DSEI 29 equipes multidisciplinares de saúde indígena (EMSI) e três Casas do Índio 9.

Os dados relativos à população indígena foram obtidos junto ao cadastro populacional registrado no Sistema de Informação da Atenção à Saúde Indígena (SIASI), no período de2001 a2007, segundo sexo e faixa etária. Os óbitos indígenas ocorridos no mesmo período foram obtidos do consolidado mensal que é encaminhado pelos pólos-base ao DSEI. No consolidado mensal são registrados todos os procedimentos realizados pela equipe multidisciplinar de saúde indígena, inclusive informações sobre a ocorrência de óbitos. O registro de óbitos em um consolidado se dá pelo fato do módulo de óbitos ainda não ter sido implantado no SIASI. Os dados de interesse desta fonte foram: idade, sexo e causas (sem codificação). Após a obtenção dos dados, os óbitos foram codificados de acordo com a 10a revisão da Classificação Internacional de Doenças (CID10). A codificação das causas de óbito e a seleção da causa básica foram realizadas por uma técnica responsável, desde 1994, pela codificação de óbitos do serviço de vigilância epidemiológica de um município com 357 mil habitantes.

Quanto à população total do estado, os dados por sexo e faixa etária, bem como os óbitos, referem-se às informações que se encontram disponíveis no site do Departamento de Informática do SUS (DATASUS; http://w3.datasus.gov.br/ datasus/datasus.php).

Os dados foram tabulados utilizando os programas Epi Info, versão 3.5.1 (Centers for Disease Control and Prevention, Atlanta, Estados Unidos), TabWin (Departamento de Informática do SUS. http://portal.saude.gov.br/portal/se/da tasus/area.cfm?id_area=732) e Microsoft Excel (Microsoft Corp., Estados Unidos) e calculados os seguintes indicadores referentes ao triênio 20042006: coeficiente geral de natalidade, coeficiente global de fecundidade, coeficiente de mortalidade infantil e seus componentes, coeficiente de mortalidade geral e por causas básicas, segundo sexo e mortalidade proporcional por causas básicas, estratificada por faixas etárias. A opção por utilizar o triênio foi no sentido de conferir maior estabilidade aos indicadores e evitar as variações aleatórias que podem ocorrer quando se tratam de eventos poucos frequentes 22 , como são os óbitos.

Para comparar a mortalidade das populações estudadas foram calculadas, em cada sexo, taxas ajustadas por idade, considerando como população padrão a população total do Estado do Mato Grosso do Sul, no sentido de se reduzir o efeito das diferenças de estrutura etária dessas populações. Após, calcularam-se a razão de mortalidade padronizada (RMP) e os respectivos intervalos de $95 \%$ de confiança (IC95\%) utilizando-se o StatCalc do aplicativo Epi Info versão 3.5.1, para avaliar os diferentes níveis de exposição. Foram consideradas significativas as diferenças entre as populações quando o IC95\% não contemplasse a unidade.

Considerando-se o fato de a população indígena corresponder a menos de $1 \%$ da população total do estado, os dados da população indígena não foram retirados da população total no cál- 
culo dos indicadores, pois não influenciaria os valores a serem assumidos. A análise dos dados envolveu o conjunto das oito etnias, compreendendo toda a população indígena do estado, uma vez que a etnia não é especificada nos registros efetuados pela FUNASA.

Para análise dos níveis de natalidade e fecundidade nas populações estudadas foram utilizados o coeficiente geral de natalidade $(\mathrm{CGN}=$ número de nascidos vivos, da área, no triênio/ população da área x 10.000) e o coeficiente de fecundidade global $(\mathrm{CFG}=$ número de nascidos vivos, da área, no período/população feminina de 15 a 49 anos da área, no período x 1.000), conforme proposto por Laurenti et al. 23. Tanto o numerador quanto o denominador das equações foram constituídos pela somatória dos dados correspondentes a cada ano do triênio.

Esta pesquisa foi aprovada pelo Comitê de Ética em Pesquisa Envolvendo Seres Humanos da Universidade Estadual de Londrina com o parecer $n^{\circ}$. 776/09, e pela Comissão Nacional de Ética em Pesquisa sob o registro no. 15.532. Por exigência do Comitê de Ética ao qual o projeto foi submetido, antecedendo o mencionado parecer, foi solicitada a Carta de Anuência à Coordenação Regional da FUNASA do Mato Grosso do Sul. A carta foi obtida mediante discussão entre a equipe técnica do Distrito Sanitário Indígena e o pesquisador principal. Após a aprovação pelos respectivos órgãos éticos, o banco de dados contendo dados do módulo demográfico do SIASI e do consolidado mensal das equipes de saúde indígena foi disponibilizado pelo Distrito Sanitário Indígena.

\section{Resultados}

Em 2007, a população indígena residente em terras indígenas no Estado do Mato Grosso do Sul compreendia 68.526 indivíduos distribuídos em 13 pólos-base e 74 terras indígenas, com ampla variação na distribuição dos contingentes populacionais por pólo-base. Os dois maiores contingentes se encontravam nos pólos-base de Amambai (com cinco terras indígenas) e Dourados (com seis terras indígenas) e concentravam 35\% da população indígena estudada.

A estrutura da pirâmide etária da população indígena difere marcadamente daquela apresentada pela população total (Figuras 1 e 2). Na população indígena, os dados segundo faixas etárias no triênio de 2004-2006 revelam maior proporção de indivíduos menores de 15 anos $(47,7 \%)$ e menor a de idosos $(5,9 \%)$, comparativamente a da população total, na qual estas foram de, respectivamente, $30,6 \%$ e $7,4 \%$.
Verificam-se também diferenças significativas nos coeficientes usados para medir natalidade. O coeficiente geral de natalidade na população indígena foi de 341,5 por 10 mil habitantes, representando 1,88 vezes (IC95\%: 1,84-1,93) o observado na população do estado, e o coeficiente de fecundidade global foi de 160,2 nascidos vivos por mil mulheres de 15-49 anos (razão de 2,45; IC95\%: 2,39-2,51). No período de 2001 a 2007, o crescimento populacional da população indígena foi de $3,15 \%$ ao ano e o da população total de 2,37\% (considerando-se os censos demográficos de 2000 e 2010; http:/ / www.ibge.gov.br).

A análise dos indicadores de saúde selecionados na população indígena revela redução de $38,8 \%$ no coeficiente mortalidade geral (de 8,5 para 5,2 por mil habitantes), $35,6 \%$ no coeficiente de mortalidade infantil (de 65,7 para 42,3 por mil nascidos vivos) e elevação discreta da proporção de óbitos de 50 anos e mais (índice de Swaroop \& Uemura passou de $28,6 \%$ para $37,3 \%$ ).

No triênio 2004-2006, o coeficiente de mortalidade geral padronizado foi significativamente mais elevado que o da população total (razão de mortalidade-RM: 1,28; IC95\%: 1,26-1,30). O valor correspondente ao índice de Swaroop \& Uemura da população indígena foi de $32 \%$ e da população total foi de $67,2 \%$. A maior proporção de óbitos indígenas se verificou entre menores de cinco anos de idade $(37,4 \%)$. O coeficiente de mortalidade infantil no triênio, na população indígena, foi de 48,9 por mil nascidos vivos, representando 2,47 (IC95\%: 2,20-2,76) vezes o verificado na população total do estado, com destaque para o componente pós-neonatal, que respondeu por $62,8 \%$ dos óbitos.

Na mortalidade por sexo e faixa etária, evidencia-se sobremortalidade masculina na população indígena em quase todas as faixas etárias, exceto nas faixas etárias de 10-14 e 55-59 anos. Ao comparar com a população total do estado, as taxas de mortalidade foram mais elevadas para a população indígena até 19 anos de idade. Após esta idade a diferença diminui, porém continuou mais elevada para a população indígena até 54 anos, apresentando redução da mortalidade a partir de 55 anos de idade.

As causas externas corresponderam à principal causa de mortalidade na população indígena, no triênio 2004-2006 (22,4\%), seguidas pelas doenças do aparelho respiratório (14,9\%), doenças do aparelho circulatório (14,3\%) e doenças infecciosas e parasitárias $(11,7 \%)$. A proporção de óbitos por causas mal definidas (12\%) na população indígena foi bem mais elevada que a da população total $(1,7 \%)$.

A importância das causas foi distinta em cada faixa etária. Em menores de um ano, 27,8\% 


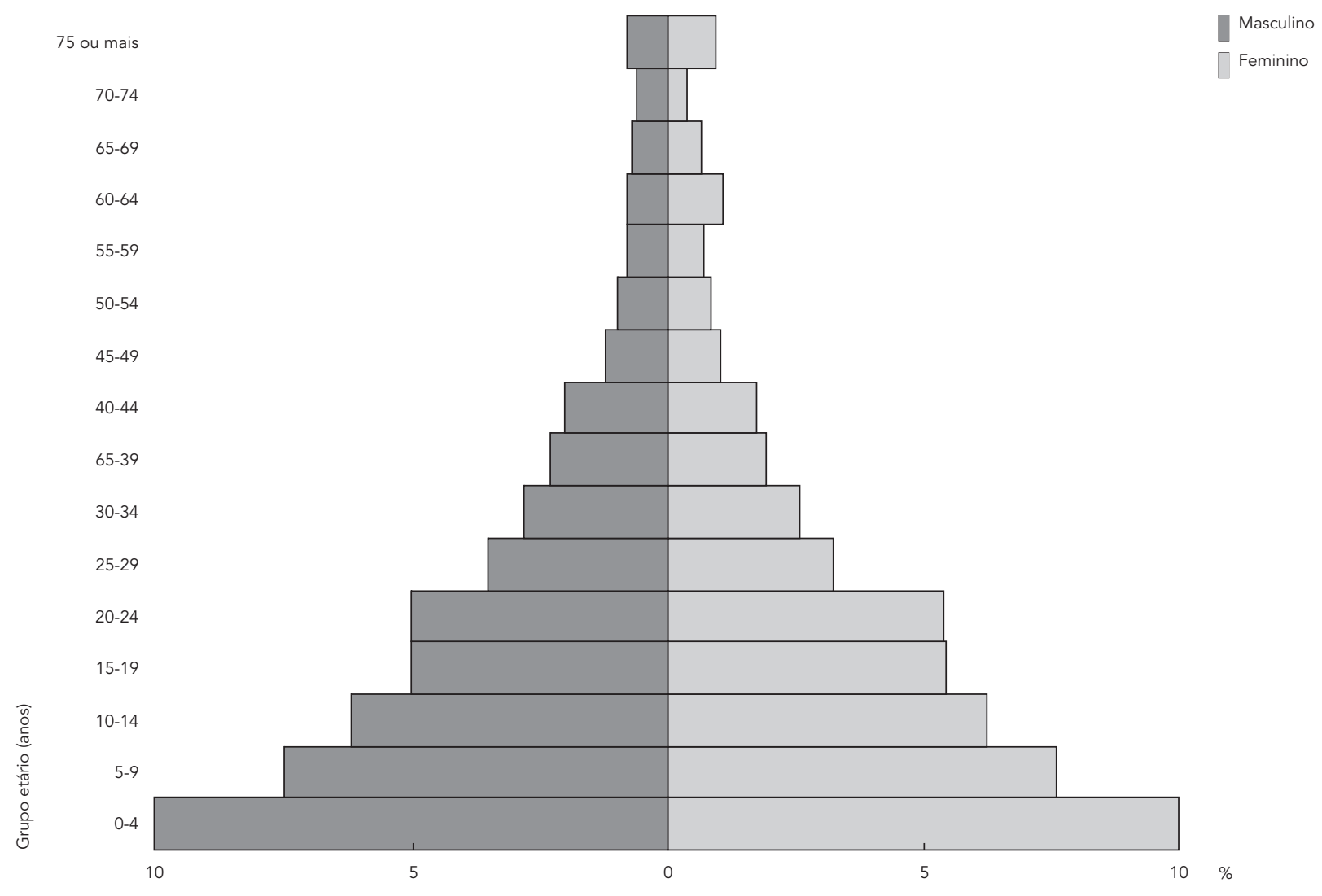

dos óbitos deveram-se às doenças do aparelho respiratório, seguidas pelas doenças infecciosas e parasitárias (13,3\%). Entre um e quatro anos, as doenças infecciosas e parasitárias foram mais importantes $(30,1 \%)$, seguidas pelas doenças do aparelho respiratório (26,9\%). Nas faixas etárias de 5 a 39 anos, as causas externas foram responsáveis por mais de $60 \%$ das mortes. A partir de 40 anos, as doenças do aparelho circulatório se destacaram e, entre os indígenas de 60 anos ou mais, responderam por $36,9 \%$ das mortes (Tabela 1 ).

O coeficiente de mortalidade por causas externas, em ambos os sexos, foi superior ao observado na população total, sendo 3,11 vezes maior (IC95\%: 2,90-3,34) entre as mulheres e 1,87 vezes maior (IC95\%: 1,81-1,94) entre os homens (Tabela 2). O maior risco de morte por causas externas na população indígena ocorreu na faixa etária de 15-19 anos e a principal causa externa foi o suicídio. A ocorrência dos suicídios na popula- ção indígena foi mais elevada entre 10 e 24 anos (Tabela 3).

Nas doenças do aparelho respiratório a população indígena apresentou taxas mais elevadas que a população total. A taxa das indígenas foi 1,20 vezes maior (IC95\%: 1,12-1,28) e entre os homens 1,47 vezes maior (IC95\%: 1,39-1,56) que a da população total. Quando se analisa por agrupamento, a influenza, a gripe e a pneumonia representaram $72,2 \%$ dos óbitos femininos e $78,6 \%$ das mortes masculinas por essas causas (Tabela 2).

Os coeficientes de mortalidade por doenças do aparelho circulatório foram significativamente menores na população indígena em ambos os sexos (RM: 0,78; IC95\%: 0,75-0,82, entre as mulheres, e RM: 0,71; IC95\%: 0,68-0,73, entre os homens). Em nível de agrupamento, a diferença foi significativa para as mulheres nas doenças cerebrovasculares (RM: 0,76; IC95\%: 0,70-0,82). Para 


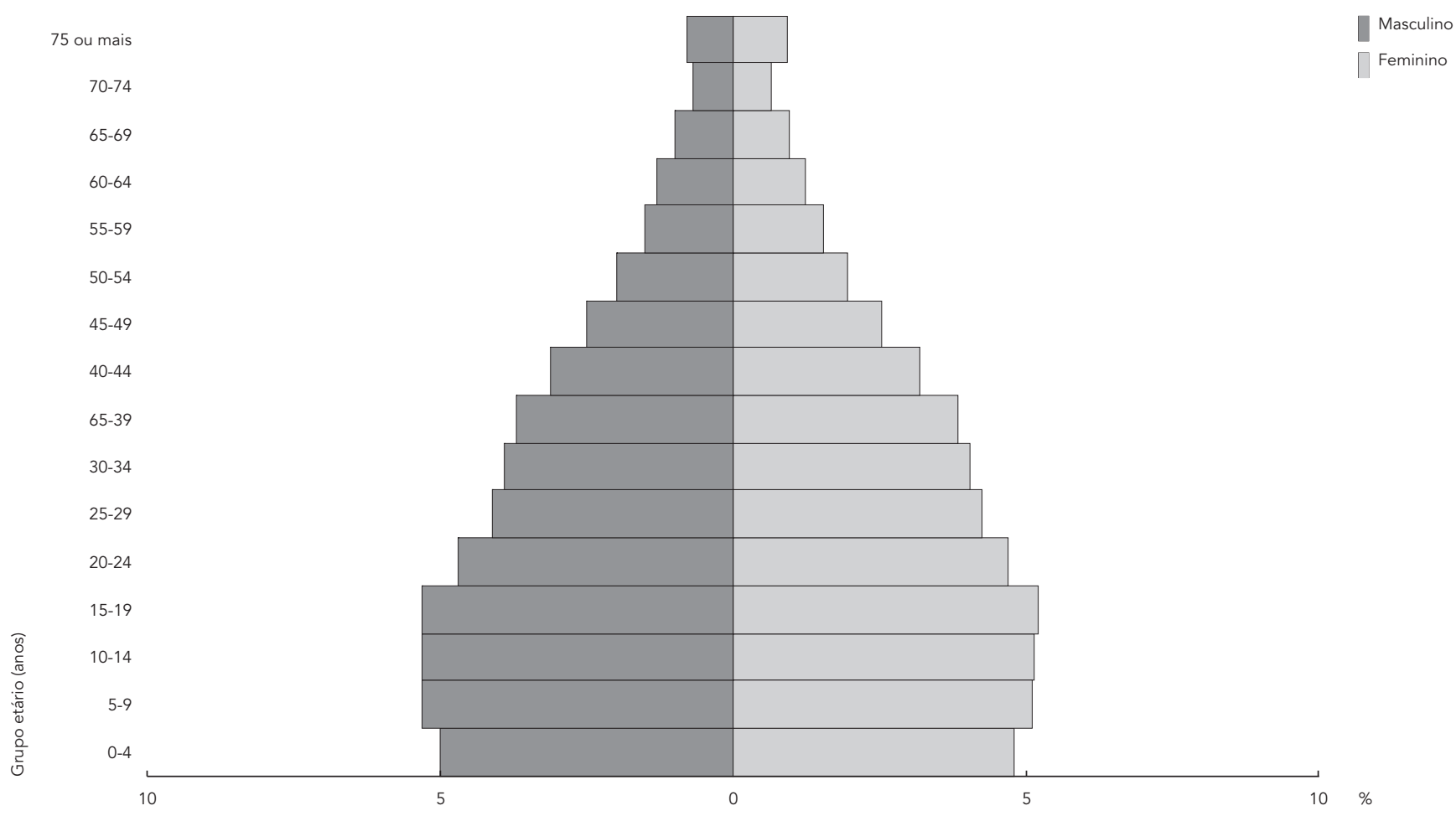

os homens as taxas foram menores nas doenças isquêmicas do coração (RM: 0,61; IC95\%: 0,580,65) e nas doenças cerebrovasculares (RM: 0,81; IC95\%: 0,76-0,87) (Tabela 2).

As doenças infecciosas e parasitárias apresentaram taxas significativamente mais elevadas para as mulheres e homens indígenas (RM: 3,15; IC95\%: 2,88-3,45 e RM: 2,44; IC95\%: 2,26-2,62, respectivamente). Quando analisadas em nível de agrupamento, mulheres e homens indígenas apresentaram maiores riscos de morte por doenças infecciosas intestinais (RM: 4,43; IC95\%: 3,795,18 e RM: 5,24; IC95\%: 4,49-6,12, respectivamente) e tuberculose (RM: 9,10; IC95\%: 6,81-12,15 e RM: 5,24; IC95\%: 4,37-6,27, respectivamente). As doenças infecciosas intestinais responderam por $64,5 \%$ das mortes deste grupo de causa entre as mulheres e $64,7 \%$ entre os homens. Para a tuberculose, estes percentuais foram de 13,1\% e 17,6\%, respectivamente (Tabela 2 ).

\section{Discussão}

Os resultados apresentados permitem verificar que as condições de saúde da população indígena são piores que as da população total. A análise dos óbitos indígenas por causas sugere estágios diferentes no processo de transição epidemiológica entre as populações de estudo. $\mathrm{Na}$ população indígena os óbitos por doenças infecciosas e parasitárias permanecem entre as causas mais importantes, ao passo que as doenças crônicas degenerativas ainda apresentam menor importância. Conforme destaca Laurenti 24 , estes resultados refletem a dinâmica da composição populacional durante longo período de tempo e resultam do comportamento da fecundidade, da mortalidade e dos deslocamentos populacionais.

Todavia, um primeiro aspecto que merece destaque refere-se à implantação da fonte de dados consultada em 1999 e, portanto, em fase de aprimoramento. Na análise dos resultados do presente estudo há necessidade de considerar as possíveis limitações, seja do ponto de vista 
Mortalidade proporcional por causas segundo faixas etárias na população indígena. Mato Grosso do Sul, Brasil, $2004-2006$.

\begin{tabular}{|c|c|c|c|c|c|c|c|c|c|c|c|c|c|c|}
\hline \multirow[t]{3}{*}{ Causas/Agrupamento * } & \multicolumn{12}{|c|}{ Faixa etária (anos) } & \multicolumn{2}{|c|}{ Total } \\
\hline & \multicolumn{2}{|c|}{$<1$} & \multicolumn{2}{|c|}{$1-4$} & \multicolumn{2}{|c|}{$5-14$} & \multicolumn{2}{|c|}{ 15-39 } & \multicolumn{2}{|c|}{ 40-59 } & \multicolumn{2}{|c|}{$60 \mathrm{ou}+$} & \multirow[b]{2}{*}{$\mathbf{n}$} & \multirow[b]{2}{*}{$\%$} \\
\hline & $\mathbf{n}$ & $\%$ & $\mathbf{n}$ & $\%$ & $\mathbf{n}$ & $\%$ & $\mathbf{n}$ & $\%$ & $\mathbf{n}$ & $\%$ & $\mathbf{n}$ & $\%$ & & \\
\hline Causas externas & 16 & 4,9 & 12 & 6,2 & 43 & 62,3 & 175 & 66,3 & 41 & 24,6 & 22 & 6,1 & 309 & 22,4 \\
\hline Acidentes de trânsito & - & - & - & - & 3 & 4,3 & 11 & 4,2 & 7 & 4,2 & 1 & 0,3 & 22 & 1,6 \\
\hline Lesões autoprovocadas & - & - & - & - & 28 & 40,6 & 92 & 34,8 & 6 & 3,6 & 1 & 0,3 & 127 & 9,2 \\
\hline Agressões & 1 & 0,3 & 2 & 1,0 & 1 & 1,4 & 33 & 12,5 & 8 & 4,8 & 2 & 0,6 & 47 & 3,4 \\
\hline $\begin{array}{l}\text { Restante das causas } \\
\text { externas }\end{array}$ & 15 & 4,6 & 10 & 5,2 & 11 & 15,9 & 39 & 14,8 & 20 & 12,0 & 18 & 5,0 & 113 & 8,2 \\
\hline $\begin{array}{l}\text { Doenças do aparelho } \\
\text { respiratório }\end{array}$ & 90 & 27,8 & 52 & 26,9 & 4 & 5,8 & 11 & 4,2 & 14 & 8,4 & 34 & 9,5 & 205 & 14,9 \\
\hline $\begin{array}{l}\text { Influenza, gripe e } \\
\text { pneumonia }\end{array}$ & 73 & 22,5 & 43 & 22,3 & 4 & 5,8 & 7 & 2,7 & 6 & 3,6 & 23 & 6,4 & 156 & 11,3 \\
\hline $\begin{array}{l}\text { Restante das doenças } \\
\text { respiratórias }\end{array}$ & 17 & 5,2 & 9 & 4,7 & - & - & 4 & 1,5 & 8 & 4,8 & 11 & 3,1 & 49 & 3,6 \\
\hline $\begin{array}{l}\text { Doenças do aparelho } \\
\text { circulatório }\end{array}$ & 3 & 0,9 & - & - & - & - & 21 & 8,0 & 40 & 24,0 & 133 & 36,9 & 197 & 14,3 \\
\hline $\begin{array}{l}\text { Doenças isquêmicas do } \\
\text { coração }\end{array}$ & - & - & - & - & - & - & 6 & 2,3 & 12 & 7,2 & 50 & 13,9 & 68 & 4,9 \\
\hline $\begin{array}{l}\text { Doenças } \\
\text { cerebrovasculares }\end{array}$ & 1 & 0,3 & - & - & - & - & 7 & 2,7 & 15 & 9,0 & 38 & 10,6 & 61 & 4,4 \\
\hline $\begin{array}{l}\text { Restante das doenças do } \\
\text { aparelho circulatório }\end{array}$ & 2 & 0,6 & - & - & - & - & 8 & 3,0 & 13 & 7,8 & 45 & 12,5 & 68 & 4,9 \\
\hline Doenças infecciosas & 43 & 13,3 & 58 & 30,1 & 7 & 10,1 & 15 & 5,7 & 9 & 5,4 & 29 & 8,1 & 161 & 11,7 \\
\hline $\begin{array}{l}\text { Doenças infecciosas } \\
\text { intestinais }\end{array}$ & 41 & 12,7 & 52 & 26,9 & 3 & 4,3 & 1 & 0,4 & - & - & 7 & 1,9 & 104 & 7,6 \\
\hline Tuberculose & - & - & - & - & - & - & 5 & 1,9 & 3 & 1,8 & 17 & 4,7 & 25 & 1,8 \\
\hline $\begin{array}{l}\text { Outras doenças } \\
\text { bacterianas }\end{array}$ & 1 & 0,3 & 4 & 2,1 & 3 & 4,3 & 1 & 0,4 & 2 & 1,2 & 4 & 1,1 & 15 & 1,1 \\
\hline $\begin{array}{l}\text { Restante das doenças } \\
\text { infecciosas }\end{array}$ & 1 & 0,3 & 2 & 1,1 & 1 & 1,4 & 8 & 3,0 & 4 & 2,4 & 1 & 0,3 & 17 & 1,2 \\
\hline Subtotal & 152 & 46,9 & 122 & 63,2 & 54 & 78,3 & 222 & 84,1 & 104 & 62,3 & 218 & 60,6 & 872 & 63,3 \\
\hline Outras causas & 172 & 53,1 & 71 & 36,8 & 15 & 21,7 & 42 & 15,9 & 63 & 37,7 & 142 & 39,4 & 505 & 36,7 \\
\hline Total & 324 & 100,0 & 193 & 100,0 & 69 & 100,0 & 264 & 100,0 & 167 & 100,0 & 360 & 100,0 & 1.377 & 100,0 \\
\hline
\end{tabular}

Fonte: consolidado mensal das equipes multidisciplinares de saúde indígena (EMSI).

* De acordo com os capítulos e agrupamentos da 10ạ revisão da Classificação Internacional de Doenças (CID-10).

qualitativo ou quantitativo, pois estudos apontam preenchimento inadequado dos dados dos SIASI, tendo em vista a diversidade, a alta rotatividade, o desconhecimento da importância do preenchimento correto e a falta de treinamento e de capacitação permanente dos profissionais que trabalham na área 25 . Souza \& Santos 26 ressaltam, ainda, a falta de uniformidade no registro de dados entre os pólos-base, duplicidade de registros, sub-registros de óbitos, registros indevidos de abortos e natimortos como nascidos vivos, registro duplicado de um natimorto também como nascido vivo.
Apesar das limitações, algumas evidências atestam favoravelmente à possibilidade de inferir sobre a situação de saúde da população indígena do estado. A proporção de $12 \%$ de mortes por causas mal definidas, um dos indicadores mais simples da qualidade de dados de mortalidade, cujos valores refletem a disponibilidade da assistência médica prestada à população e as características do preenchimento da declaração de óbito, foi inferior a 13,3\% registrada para o país em 2003 27, o que sugere a viabilidade de utilização da fonte consultada. 
Proporção de óbitos, coeficiente de mortalidade padronizado (por 100 mil habitantes) por causas, segundo sexo, na população indígena e total do estado, razão de mortalidade padronizada (RMP) e respectivo intervalo de 95\% de confiança (IC95\%). Estado do Mato Grosso do Sul, Brasil, $2004-2006$.

\begin{tabular}{|c|c|c|c|c|c|c|c|}
\hline \multirow[t]{2}{*}{ Causas/Agrupamento * } & \multicolumn{3}{|c|}{ Indígenas ** } & \multicolumn{3}{|c|}{ População total *** } & \multirow[t]{2}{*}{ RMP (IC95\%) } \\
\hline & $\mathrm{n}$ & $\%$ & Coeficiente & $\mathbf{N}$ & $\%$ & Coeficiente & \\
\hline \multicolumn{8}{|l|}{ Feminino } \\
\hline Causas externas & 86 & 15,0 & 93,4 & 1.015 & 6,9 & 30,0 & $3,11(2,90-3,34)$ \\
\hline Acidentes de trânsito & 7 & 8,1 & 8,9 & 423 & 41,7 & 12,5 & $0,71(0,61-0,82)$ \\
\hline Lesões autoprovocadas & 37 & 43,0 & 38,0 & 133 & 13,1 & 3,9 & $9,64(8,06-11,52)$ \\
\hline Agressões & 9 & 10,5 & 12,2 & 180 & 17,7 & 5,3 & $2,28(1,92-2,72)$ \\
\hline Restante das causas externas & 33 & 38,4 & 34,8 & 279 & 27,5 & 8,3 & $4,22(3,70-4,81)$ \\
\hline Doenças do aparelho respiratório & 79 & 15,0 & 57,2 & 1.607 & 6,9 & 47,6 & $1,20(1,12-1,28)$ \\
\hline Influenza, gripe e pneumonia & 57 & 72,2 & 34,3 & 707 & 44,0 & 20,9 & $1,64(1,49-1,80)$ \\
\hline Restante das doenças respiratórias & 22 & 27,8 & 23,1 & 900 & 56,0 & 26,6 & $0,87(0,79-0,96)$ \\
\hline Doenças do aparelho circulatório & 86 & 15,0 & 118,0 & 5.101 & 34,8 & 151,0 & $0,78(0,75-0,82)$ \\
\hline Doenças isquêmicas do coração & 33 & 38,4 & 47,3 & 1.525 & 29,9 & 45,1 & $1,05(0,98-1,12)$ \\
\hline Doenças cerebrovasculares & 24 & 27,9 & 36,1 & 1.607 & 31,5 & 47,6 & $0,76(0,70-0,82)$ \\
\hline Restante das doenças circulatórias & 29 & 33,7 & 40,6 & 1.969 & 38,6 & 58,3 & $0,70(0,65-0,75)$ \\
\hline Doenças infecciosas e parasitárias & 76 & 13,3 & 59,6 & 638 & 4,4 & 18,9 & $3,15(2,88-3,45)$ \\
\hline Doenças infecciosas intestinais & 49 & 64,5 & 25,2 & 192 & 30,1 & 5,7 & $4,43(3,79-5,18)$ \\
\hline Tuberculose & 10 & 13,1 & 13,7 & 51 & 8,0 & 1,5 & $9,10(6,81-12,15)$ \\
\hline Outras doenças bacterianas & 11 & 14,5 & 11,7 & 163 & 25,5 & 4,8 & $2,42(2,02-2,91)$ \\
\hline Restante das doenças infecciosas & 6 & 7,9 & 8,7 & 232 & 36,4 & 6,9 & $1,27(1,07-1,51)$ \\
\hline \multicolumn{8}{|l|}{ Masculino } \\
\hline Causas externas & 223 & 27,7 & 268,2 & 4.839 & 20,9 & 143,1 & $1,87(1,81-1,94)$ \\
\hline Acidentes de trânsito & 15 & 6,7 & 21,5 & 1.660 & 34,3 & 49,1 & $0,44(0,40-0,48)$ \\
\hline Lesões autoprovocadas & 90 & 40,4 & 102,5 & 447 & 9,2 & 13,2 & $7,76(7,03-8,56)$ \\
\hline Agressões & 38 & 17,0 & 51,0 & 1.776 & 36,7 & 52,5 & $0,97(0,91-1,04)$ \\
\hline Restante das causas externas & 80 & 35,9 & 92,2 & 956 & 19,8 & 28,3 & $3,26(3,03-3,51)$ \\
\hline Doenças do aparelho respiratório & 126 & 15,7 & 88,7 & 2.039 & 8,8 & 60,0 & $1,47(1,39-1,56)$ \\
\hline Influenza, gripe e pneumonia & 99 & 78,6 & 65,2 & 905 & 44,4 & 26,8 & $2,44(2,26-2,63)$ \\
\hline Restante das doenças respiratórias & 27 & 21,4 & 23,5 & 1.134 & 55,6 & 33,5 & $0,70(0,64-0,77)$ \\
\hline Doenças do aparelho circulatório & 111 & 13,8 & 148,4 & 7.090 & 30,6 & 209,6 & $0,71(0,68-0,73)$ \\
\hline Doenças isquêmicas do coração & 35 & 31,5 & 48,5 & 2.675 & 37,7 & 79,1 & $0,61(0,58-0,65)$ \\
\hline Doenças cerebrovasculares & 37 & 33,3 & 47,6 & 1.978 & 27,9 & 58,5 & $0,81(0,76-0,87)$ \\
\hline Restante das doenças circulatórias & 39 & 35,1 & 52,1 & 2.437 & 34,4 & 72,0 & $0,72(0,68-0,77)$ \\
\hline Doenças infecciosas e parasitárias & 85 & 10,6 & 71,1 & 987 & 4,4 & 29,2 & $2,44(2,26-2,62)$ \\
\hline Doenças infecciosas intestinais & 55 & 64,7 & 29,4 & 190 & 19,3 & 5,6 & $5,24(4,49-6,12)$ \\
\hline Tuberculose & 15 & 17,6 & 21,7 & 140 & 14,2 & 4,1 & $5,24(4,37-6,27)$ \\
\hline Outras doenças bacterianas & 4 & 4,7 & 3,0 & 173 & 17,5 & 5,1 & $0,58(0,46-0,75)$ \\
\hline Restante das doenças infecciosas & 11 & 13,0 & 14,2 & 484 & 49,0 & 14,3 & $1,00(0,88-1,13)$ \\
\hline
\end{tabular}

* De acordo com os capítulos e agrupamentos da 10ª revisão da Classificação Internacional de Doenças (CID-10);

** De acordo com o consolidado mensal das equipes multidisciplinares de saúde indígena (EMSI);

*** Dados do Sistema de Informações sobre Mortalidade (SIM; http://www.datasus.gov.br).

Relativamente à influência dos deslocamentos na composição da população indígena do Estado do Mato Grosso do Sul, poucos estudos registram a ocorrência do fenômeno migratório nesta população. Atualmente, há relato de deslocamentos de terras indígenas para os centros urbanos, em busca de melhores condições de vida 12 . No entanto, estima-se que em torno de 3 mil índios estão dispersos nas áreas urbanas, quantidade que não influencia sobremaneira a estrutura demográfica aqui verificada. 
Número de óbitos e coeficiente de mortalidade por suicídio (por 100 mil habitantes) para mulheres, homens e população total indígena segundo faixa etária. Estado do Mato Grosso do Sul, Brasil, 2004-2006.

\begin{tabular}{|c|c|c|c|c|c|c|}
\hline \multirow[t]{2}{*}{ Faixa etária (anos) } & \multicolumn{2}{|c|}{ Feminino } & \multicolumn{2}{|c|}{ Masculino } & \multicolumn{2}{|c|}{ Total } \\
\hline & $\mathrm{n}$ & Coeficiente & $\mathrm{n}$ & Coeficiente & $\mathrm{n}$ & Coeficiente \\
\hline$<5$ & - & - & - & - & - & - \\
\hline $5-9$ & - & - & - & 13,9 & 2 & 6,9 \\
\hline $10-14$ & 19 & 157,7 & 7 & 58,9 & 26 & 108,6 \\
\hline $15-19$ & 11 & 105,0 & 33 & 339,1 & 44 & 217,7 \\
\hline $20-24$ & 1 & 9,7 & 23 & 238,9 & 24 & 120,1 \\
\hline $25-29$ & 3 & 47,9 & 10 & 148,5 & 10 & 100,0 \\
\hline $30-34$ & 1 & 20,2 & 8 & 147,5 & 9 & 86,7 \\
\hline $35-39$ & - & - & 2 & 48,1 & 2 & 25,4 \\
\hline $40-44$ & 1 & 29,6 & 1 & 26,5 & 2 & 28,0 \\
\hline $45-49$ & - & - & 1 & 41,8 & 1 & 23,0 \\
\hline $50-54$ & 1 & 61,1 & 1 & 54,5 & 2 & 57,6 \\
\hline $55-59$ & - & - & 1 & 65,6 & 1 & 34,5 \\
\hline $60-64$ & - & - & - & - & - & - \\
\hline $65-69$ & - & - & 1 & 75,0 & 1 & 38,9 \\
\hline 70 ou + & - & - & - & - & - & - \\
\hline Total & 37 & 38,6 & 90 & 92,9 & 127 & 65,9 \\
\hline
\end{tabular}

Fonte: consolidado mensal das equipes multidisciplinares de saúde indígena (EMSI).

Assim, a estrutura da pirâmide etária indígena, com base larga e ápice estreito, reflete as altas taxas de natalidade e de mortalidade nos primeiros anos de vida, revelando atraso na transição demográfica da população indígena comparativamente à do estado. A forma da pirâmide da população indígena é semelhante à do Brasil na década de 1950, que entre as décadas de 1960 e 2000, com a redução da fecundidade e da mortalidade, teve a pirâmide com sua forma tradicional modificada. No ano 2000, a estrutura etária do país já refletia a redução da população jovem e o crescimento do número de idosos 28.

Vários estudos demográficos indicam a composição tipicamente jovem em populações indígenas, com proporções de menores de 15 anos, variando de $44,5 \%$ a $56,2 \%$ 5,6,29,30,31,32. Estas proporções são determinadas pelas taxas de natalidade que superam as de mortalidade e promovem o crescimento populacional. As taxas apresentadas pela população indígena em estudo acompanham a tendência observada em outras populações, caracterizadas por altas taxas de natalidade e fecundidade, com consequente crescimento anual de $3 \%$ a $8,4 \%$ 4,5,29,31,32.

Apesar de a análise ter se limitado à taxa de natalidade e à de fecundidade global, os valores observados são sugestivos da alta taxa de fecundidade total (filhos por mulher), como observada no povo Xavante de Sangradouro Volta Grande (Mato Grosso), cuja alta taxa de fecundidade total (8,6 filhos por mulher) explica-se pelo início precoce da vida reprodutiva, que ocorre por volta de 13 anos de idade e se prolonga até os 45 anos de idade, com intervalo interpartal de 23,3 meses 29 . Além disso, altas taxas de fecundidade também estão relacionadas a fatores socioculturais como a valorização de famílias numerosas. Camargo et al. 33 constataram que o aumento do número médio de filhos por mulher no povo Kamaiurá estava relacionado com a intenção de expandir suas famílias para ampliar seu destaque no cenário político do Alto Xingu.

O padrão de mortalidade caracterizado por elevada proporção de mortes precoces na população indígena, com maior concentração dos óbitos em menores de cinco anos, pode estar expressando precárias condições de vida, com alta prevalência de desnutrição infantil e infecções associadas. Esta situação foi verificada na população Xavante de Mato Grosso, na qual 77,7\% de todos os óbitos se concentraram nos primeiros cinco anos de vida 5. Evidenciam, ainda, as condições precárias de saúde desta população, a elevada taxa de mortalidade infantil, com seu componente de maior peso o pós-neonatal, cujas principais causas de óbitos, como as doenças do aparelho respiratório e as infecciosas e parasitá- 
rias são passíveis de prevenção com a utilização de tecnologias simples e de custo relativamente baixo ${ }^{34}$. Quando comparada à situação da população total do estado e da população brasileira em geral, no mesmo período, aponta ainda a desigualdade na situação sanitária, ambiental e de assistência à saúde. O coeficiente de mortalidade infantil da população total do estado, no triênio analisado, foi de 19,8 por mil nascidos vivos e da população brasileira em geral, de 17,1. Em ambas, o componente principal foi o neonatal: 12,8 e 11,4 por mil nascidos vivos, respectivamente (DATASUS. http://w3.datasus.gov.br/datasus/ datasus.php). Esta situação pode ser em parte atribuída a deficiências na assistência à saúde prestada, mas provavelmente relaciona-se também às precárias condições ambientais e de nutrição desta população 13,14.

Na literatura não há nenhuma explicação para a redução da mortalidade após os 55 anos de idade verificada no nosso estudo. Esta situação poderia ser explicada a partir da qualidade dos dados e possível superestimação da idade por parte dos indivíduos dessa faixa etária, como foi sugerido por Souza et al. 5. Na época em que estes indivíduos nasceram não havia registros de nascimentos, sem mencionar que as linguagens matemáticas dos povos indígenas ainda são pouco conhecidas e que cada povo indígena possui sua forma de classificar a idade 35 .

Apesar da pior situação observada entre os indígenas, o aumento na proporção de mortes em indivíduos com 50 anos ou mais (índice de Swaroop \& Uemura de $32 \%$ ), a redução do coeficiente de mortalidade geral e da mortalidade infantil, no período de 2001 a 2007, aponta melhorias nas condições de saúde, que pode ser consequente à atuação da FUNASA que, desde 1999, vem prestando assistência à saúde e ampliando as ações de saneamento básico nas aldeias. No entanto, a mortalidade por causas difere da população brasileira em geral, cujas principais causas de óbitos foram as doenças cardiovasculares, seguidas pelas neoplasias e causas externas (DATASUS. http://w3.datasus.gov.br/datasus/datasus.php), bem como dos resultados relatados para a população indígena do Estado de Pernambuco, na qual as causas mal definidas, por doenças do aparelho circulatório, causas externas e neoplasias foram as principais causas de óbitos 36 .

O elevado percentual de óbitos por doenças infecciosas e parasitárias na população indígena indica um contraste entre estes povos e a população brasileira, cujos óbitos por essas doenças vêm declinando desde a década de 1930 28. Uma proporção de doenças infecciosas intestinais, semelhante à apresentada pela população indígena estudada, era observada no país antes da década de 1980 (DATASUS. http://w3.datasus.gov.br/da tasus/datasus.php). Posteriormente, em 2008, já se apresentava em $5 \%$.

As doenças intestinais, principalmente a diarreia, são comuns em áreas com precárias condições de saneamento 37. A qualidade da água, mudança nos hábitos de higiene, assim como a adequação do esgotamento sanitário, foram fatores importantes no controle dessas doenças na população brasileira 38 . A educação em saúde e a terapia de reidratação oral também contribuíram significativamente para a diminuição da mortalidade por essas doenças 38. No ano 2003, um estudo conduzido com a população Xavante no Estado do Mato Grosso evidenciou a precariedade nas aldeias, caracterizada pela falta de coleta de lixo e inadequado destino dos dejetos. A água encanada para consumo era proveniente de córregos onde eram lavadas as roupas e utensílios. Embora os adultos andassem calçados, as crianças não o faziam 7 .

O maior risco de morte por tuberculose observado na população indígena estudada pode estar relacionado ao confinamento e à condição nutricional deste grupo populacional, pois se sabe que esta doença ocorre com maior frequência em regiões com precárias condições sociossanitárias e atinge os indivíduos mais frágeis do ponto de vista imunológico. Grande parte desta fragilidade está associada às deficiências nutricionais. Vários estudos evidenciaram as precárias condições nutricionais na população indígena do Estado do Mato Grosso do Sul 9,13,14. Em um estudo conduzido com índios Panará, do sul do Pará, foi verificado que a elevada prevalência de tuberculose estava associada à habitação pouco ensolarada, sem divisões internas, e ao excessivo número de moradores na mesma residência 39 . É bastante provável que estas condições e a carência nutricional também expliquem os óbitos por doenças do aparelho respiratório.

Paradoxalmente, a persistência das doenças infecciosas e parasitárias, nota-se também na população indígena estudada a importância relativa das doenças cardiovasculares, relacionadas às mudanças nos padrões comportamentais. Apesar da menor importância dessas doenças na população indígena estudada, é possível aventar a hipótese de alterações no estilo de vida, principalmente dieta e atividade física. Ainda que em menor intensidade do que na população total, a semelhança nas taxas de mortalidade entre as mulheres indígenas e não indígenas sugere alterações em curso no padrão alimentar, com maior consumo de alimentos industrializados. Atualmente, na população indígena do Estado de Pernambuco, já se verifica tal impacto, pois as doenças crônicas e degenerativas se apresentam 
como principais causas de óbitos, ao passo que as doenças infecciosas e parasitárias ocupam o último lugar em importância 36 . Vários autores evidenciaram alterações metabólicas e antropométricas em populações indígenas, relacionadas às mudanças nos padrões alimentares e atividades físicas 40,41 .

Outra causa relacionada às mudanças nos padrões de mortalidade refere-se às altas taxas de mortes por causas externas. Há uma forte relação entre alcoolismo e morte por causas externas nessas populações. Em muitas comunidades indígenas, o álcool foi introduzido pelos próprios colonizadores como arma de dominação e estudos mostram elevada prevalência de uso abusivo e até mesmo de dependência nesta população ${ }^{3}$.

Ainda em relação às causas externas, chamam atenção as altas taxas de suicídio na população indígena, já constatadas também em outras populações 42,43 . A prática do suicídio tem sido atribuída, por alguns autores, à destruição da cultura causada pela perda de seus antigos territórios e aos problemas gerados pelo confinamento compulsório em pequenas áreas de terra em que se encontram atualmente 20 . A alta taxa de mortalidade de jovens por suicídio é um problema que vem se agravando ao longo do tempo em comunidades indígenas. Na população indígena Kaiowá foi verificada uma taxa 40 vezes maior que a brasileira, mas é entre os Suruwahá do Estado do Amazonas que a situação é pior, com uma taxa estimada de 1.922 casos por 100 mil habitantes ${ }^{42}$, sendo mais precoce em mulheres (12 a 17 anos) 43 . Esta prática, segundo historiadores, é recente no povo Suruwahá e teve sua origem no extermínio ocasionado pelo confronto acentuado com os colonizadores 44 .

Em relação ao Estado do Mato Grosso do Sul, a pior situação é verificada entre os Kaiowá/ Guarani e é relacionada ao confinamento compulsório em áreas reduzidas de terras. A quantidade de terras da qual dispõem, atualmente, não oferece condições de sobrevivência física (menos de 1 hectare por família). Em Dourados (Mato Grosso do Sul), onde a situação dos suicídios é mais crítica, há 13 mil índios distri- buídos em uma área de 3,6 mil hectares, que é atravessada por várias rodovias e ocupada pelas aldeias indígenas, o que torna a terra disponível absolutamente insuficiente para a produção agrícola ${ }^{45}$. Além disso, discute-se a dificuldade da manutenção da antiga organização social e religiosa 10 com a quantidade reduzida de terras. Os impasses na ampliação dos territórios, os conflitos interétnicos, o preconceito, a interferência excessiva de vários órgãos externos e o impacto desestruturante do trabalho assalariado nas usinas de álcool e fazendas de gado têm contribuído para o aumento do clima de desespero entre essa população 20 . Aliado a isso, ainda há os conflitos internos relacionados à disputa pelo domínio do espaço e autoridade política que têm causado a quebra dos relacionamentos familiares e afetivos e consequentemente levado ao suicídio 10,20 .

Apesar das limitações do presente estudo, como a impossibilidade de se considerar a diversidade sociocultural das oito etnias a partir da fonte de dados utilizada e a possível falta de uniformidade no preenchimento dos formulários relativos aos óbitos entre os profissionais envolvidos, os resultados sugerem, em certa medida, qualidade aceitável dos dados, diferentemente da situação descrita por outros autores que identificaram alta proporção de óbitos por causas mal definidas em populações indígenas 5,40 .

Cabe assim destacar que a melhoria do acesso às ações assistenciais com a criação do subsistema de saúde específico para esta população constitui importante avanço na assistência à saúde indígena e nossos resultados indicam melhorias dos principais indicadores de saúde, entre 2001 e 2007, com redução de 38,8\% do coeficiente de mortalidade geral, $35,6 \%$ do coeficiente de mortalidade infantil e aumento da proporção de óbitos a partir de 50 anos de idade. Na continuidade do processo, há que se implementar efetivamente as políticas públicas inclusivas na redução das desigualdades observadas entre as populações estudadas e a importância da qualificação dos sistemas de informação, como as utilizadas, no processo de acompanhamento. 


\section{Resumo}

O objetivo do estudo foi o de analisar os aspectos demográficos e o padrão de mortalidade da população indígena aldeada do Estado do Mato Grosso do Sul, Brasil, comparativamente ao da população total do estado. Foram calculados indicadores de mortalidade a partir dos dados obtidos do Sistema de Informação de Atenção à Saúde Indígena e do consolidado mensal, assim como, do módulo demográfico e do Sistema de Informações sobre Mortalidade do SUS. Observaramse, na população indígena, comparativamente à do estado, maior proporção de indivíduos menores de 15 anos e menor de idosos e taxas mais elevadas de mortalidade em idades precoces e por doenças infecciosas e parasitárias. Os homens indígenas apresentaram taxas significativamente maiores para as causas externas, doenças do aparelho respiratório e doenças infecciosas. Entre as mulheres, apenas as causas externas e doenças infecciosas se destacaram. A grande importância dos suicídios na juventude apresentou-se como aspecto relevante. As condições de saúde da população indígena são piores que a da população total.

Índios Sul-Americanos; População Indígena; Indicadores Básicos de Saúde; Desigualdades em Saúde

\section{Colaboradores}

M. E. V. Ferreira elaborou o estudo, coletou informações, analisou e redigiu o manuscrito. T. Matsuo e R. K. T. Souza participaram na concepção da pesquisa, estruturação do artigo, discussão dos resultados e revisão crítica do texto.

\section{Agradecimentos}

Ao Distrito Sanitário Especial Indígena de Campo Grande, Mato Grosso do Sul, pela disponibilização dos dados utilizados nesta pesquisa.

\section{Referências}

1. Santos RV, Coimbra Jr. CEA. Cenários e tendências da saúde e da epidemiologia dos povos indígenas no Brasil. In: Coimbra Jr. CEA, Santos RV, Escobar AL, organizadores. Epidemiologia e saúde dos povos indígenas. Rio de Janeiro: Editora Fiocruz/ ABRASCO; 2003. p. 13-48.

2. Schmidt MI, Duncan BB, Silva GA, Menezes AM, Monteiro CA, Barreto SM, et al. Chronic non-communicable diseases in Brazil: burden and current challenges. Lancet 2011; 377:1949-61.

3. Guimarães LAM, Grubits S. Alcoolismo e violência em etnias indígenas: uma visão crítica da situação brasileira. Psicol Soc 2007; 19:45-51.

4. Machado M, Pagliaro H, Baruzzi RG. Perfil demográfico dos Hupd'äh, povo Maku da região do Alto Rio Negro, Amazonas (2000-2003). Rev Bras Estud Popul 2009; 26:37-50.
5. Souza LG, Santos RV, Coimbra Jr. CEA. Estrutura etária, natalidade e mortalidade do povo indígena Xavante de Mato Grosso, Amazônia, Brasil. Ciênc Saúde Coletiva 2010; 15 Suppl 1:1465-73.

6. Pagliaro H. A revolução demográfica dos povos indígenas no Brasil: a experiência dos Kayabí do Parque Indígena do Xingu, Mato Grosso, Brasil, 1970-2007. Cad Saúde Pública 2010; 26:579-90.

7. Leite MS, Gugelmin SA, Santos RV, Coimbra Jr. CEA. Perfis de saúde indígena, tendências nacionais e contextos locais: reflexões a partir do caso Xavante, Mato Grosso. In: Coimbra Jr. CEA, Santos $\mathrm{RV}$, Escobar AL, organizadores. Epidemiologia e saúde dos povos indígenas. Rio de Janeiro: Editora Fiocruz/ABRASCO; 2003. p. 105-26. 
8. Brasil. Portaria $\mathrm{n}^{\circ}$. $1163 / \mathrm{GM}$, de 14 de setembro de 1999. Dispõe sobre as responsabilidades na prestação de assistência à saúde dos povos indígenas, no Ministério da Saúde e dá outras providências. Diário Oficial, Brasília 1999; 15 set.

9. Coordenação Regional do Mato Grosso do Sul, Fundação Nacional de Saúde. Relatório de atividades desenvolvidas na saúde indígena. Campo Grande: Fundação Nacional de Saúde; 2007.

10. Brand A. Os complexos caminhos da luta pela terra entre os Kaiowá e Guarani no MS. Tellus 2004; 4:137-50.

11. Oliveira JE, Pereira LM. Duas no pé e uma na bunda: da participação Terena na guerra entre o Paraguai e a Tríplice Aliança à luta pela ampliação dos limites da terra indígena Buriti. Revista História em Reflexão 2007; 1:1-19.

12. Santana GR. Uma análise do processo migratório dos índios Terena para o perímetro urbano da cidade. Revista de Iniciação Científica da FFC 2004; 4:19-34.

13. Ribas DL, Philippi ST. Aspectos alimentares e nutricionais de mães e crianças indígenas Terena, Mato Grosso do Sul. In: Coimbra Jr. CEA, Santos RV, Escobar AL, organizadores. Epidemiologia e saúde dos povos indígenas. Rio de Janeiro: Editora Fiocruz/ABRASCO; 2003. p. 73-88.

14. Morais MB, Alves GMS, Fagundes-Neto U. Estado nutricional de crianças índias Terenas: evolução do peso e estatura e prevalência atual de anemia. J Pediatr (Rio J.) 2005; 81:383-9.

15. Ribas DLB, Zorzatto JR, Saad MNL, Philippi ST. Saúde e doença em crianças indígenas Terena. Cad Saúde Colet (Rio J.) 2001; 9:139-54.

16. Pícoli RP, Carandina L, Ribas DLB. Saúde maternoinfantil e nutrição de crianças Kaiowá e Guarani, Área Indígena de Caarapó, Mato Grosso do Sul, Brasil. Cad Saúde Pública 2006; 22:223-7.

17. Aguiar JI. Enfermidades degenerativas entre os Terena de Mato Grosso do Sul: uma abordagem de aspectos ligados ao diabetes tipo II e fatores correlacionados. In: Anais do Seminário sobre Alcoolismo e DST/AIDS entre os Povos Indígenas da Macrorregião Sul, Sudeste e Mato Grosso do Sul. Brasília: Ministério da Saúde; 2001. p. 77-82.

18. Aguiar JI. Alcoolismo em população Terena no Estado de Mato Grosso do Sul: impacto da sociedade envolvente. In: Anais do Seminário sobre Alcoolismo e DST/AIDS entre os Povos Indígenas da Macrorregião Sul, Sudeste e Mato Grosso do Sul. Brasília: Ministério da Saúde; 2001. p. 146-66.

19. Braga-Neto JA, Parizotto SPCOL, Francischinelli FCP, Moraes TS, Nantes ARF. Levantamento do estado de saúde de menores de seis anos da reserva indígena Kaiowá/Guarani de Caarapó-MS. Tellus 2001; 1:103-16.

20. Brand A, Vietta K. Análise gráfica das ocorrências de suicídios entre os Kaiowá/Guarani, no Mato Grosso do Sul, entre 1981 e 2000. Tellus 2001; 1:119-32.
21. Foti MV. A morte por jejuvy entre os Guarani do sudoeste brasileiro. Revista de Estudos e Pesquisas 2004; 1:45-72.

22. Pereira MG. Epidemiologia: teoria e prática. Rio de Janeiro: Editora Guanabara Koogan; 1995.

23. Laurenti R, Jorge MHPM, Lebrão ML, Gotlieb SLD. Estatísticas de saúde. São Paulo: EPU; 2005.

24. Laurenti R. Transição demográfica e transição epidemiológica. In: Anais do Congresso Brasileiro de Epidemiologia, 1990. Campinas: Universidade Estadual de Campinas; 1990. p. 143-5.

25. Sousa MC, Scatena JHG, Santos RV. O Sistema de Informação da Atenção à Saúde Indígena (SIASI): criação, estrutura e funcionamento. Cad Saúde Pública 2007; 23:853-61.

26. Souza LG, Santos RV. Componente demográfico do Sistema de Informação da Atenção à Saúde Indígena, DSEI Xavante, Mato Grosso, Brasil. Caderno CRH 2009; 57:523-9.

27. Santo AH. Causas mal definidas de morte e óbitos sem assistência. Rev Assoc Méd Bras 2008; 54:23-8.

28. Vermelho LL, Monteiro MFG. Transição demográfica e epidemiológica. In: Medronho RA, Bloch KV, Luiz RR, Werneck GL, organizadores. Epidemiologia. São Paulo: Editora Atheneu; 2003. p. 91-103.

29. Souza LG, Santos RV. Perfil demográfico da população indígena Xavánte de Sangradouro-Volta Grande, Mato Grosso (1993-1997), Brasil. Cad Saúde Pública 2001; 17:355-65.

30. Pagliaro H, Carvalho NS, Rodrigues D, Baruzzi RG. Demographic dynamics of the Suyá, a Jê people of the Xingu Indigenous Park, Central Brazil, 19702004. Cad Saúde Pública 2007; 23:1071-81.

31. Pagliaro H, Junqueira C, Mendaña LGS, Mendonça SB, Baruzzi RG. Dinâmica demográfica dos Kamaiurá, povo Tupi do Parque Indígena do Xingu, Mato grosso, Brasil, 1970-1999. Rev Bras Estud Popul 2008; 25:377-88.

32. Souza LG, Pagliaro H, Santos RV. Perfil demográfico dos índios Bororó de Mato Grosso, Brasil, 19931996. Cad Saúde Pública 2009; 25:328-36.

33. Camargo CPF, Junqueira C, Pagliaro H. Reflexões acerca do mundo cultural e do comportamento reprodutivo dos Kamaiúra ontem e hoje. In: Pagliaro H, Azevedo MM, Santos RV, organizadores. Demografia dos povos indígenas no Brasil. Rio de Janeiro: Editora Fiocruz/Associação Brasileira de Estudos Populacionais; 2005. p. 119-34.

34. Correia LL, McAuliffe JF. Saúde materno-infantil. In: Rouquayrol MZ, Almeida Filho N, organizadores. Epidemiologia e saúde. 6a Ed. Rio de Janeiro: Editora Guanabara Koogan; 2003. p. 375-404.

35. Pagliaro H, Azevedo MM, Santos RV. Demografia dos povos indígenas no Brasil: um panorama crítico. In: Pagliaro H, Azevedo MM, Santos RV, organizadores. Demografia dos povos indígenas no Brasil. Rio de Janeiro: Editora Fiocruz/Associação Brasileira de Estudos Populacionais; 2005. p. 11-32. 
36. Costa AM, Gonçalves GMS, Lima TFP, Lima QSS. Epidemiologia e determinantes das DCNT em populações indígenas no Brasil. In: Freese E, organizador. Epidemiologia, políticas e determinantes das doenças crônicas não transmissíveis no Brasil. Recife: Editora Universitária da UFPE; 2006. p. 303-20.

37. Coimbra Jr. CEA, Santos RV. Saúde indígena. In: Comissão Nacional Sobre Determinantes Sociais da Saúde, organizadores. As causas sociais das iniquidades em saúde no Brasil. Rio de Janeiro: Editora Fiocruz; 2008. p. 128-30.

38. Departamento de Vigilância Epidemiológica, Secretaria de Vigilância em Saúde, Ministério da Saúde. Doenças infecciosas e parasitárias: manual de bolso. Brasília: Ministério da Saúde; 2006.

39. Baruzzi RG, Barros VL, Rodrigues D, Souza ALM, Pagliaro H. Saúde e doença em índios Panará (Kreen-Akarôre) após vinte e cinco anos de contato com o nosso mundo, com ênfase na ocorrência de tuberculose (Brasil Central). Cad Saúde Pública 2001; 17:407-12.
40. Cardoso AM, Mattos IE, Koifman RJ. Prevalência de fatores de risco para doenças cardiovasculares na população Guaraní-Mbyá do Estado do Rio de Janeiro. Cad Saúde Pública 2001; 17:345-54.

41. Salvo VLMA, Rodrigues D, Baruzzi RG, Pagliaro H, Gimeno SGA. Perfil metabólico e antropométrico dos Suyá. Parque Indígena do Xingu, Brasil Central. Rev Bras Epidemiol 2009; 12:458-68.

42. Oliveira CS, Lotufo-Neto F. Suicídio entre povos indígenas: um panorama estatístico brasileiro. Rev Psiquiatr Clín 2003; 30:4-10.

43. Poz JD. Crônica de uma morte anunciada. Rev Antropol (São Paulo) 2000; 43:89-144.

44. Souza KT, Santos MM. Morte ritual: reflexões sobre o "suicídio" Suruwahá. Espaço Ameríndio 2009; 3:10-24.

45. Brand A. Índios precisam produzir seus alimentos. http://www.tekoarandu.org/ (acessado em 10/ Jan/ 2010).

Recebido em 10/Fev/2011

Versão final reapresentada em 14/Ago/2011

Aprovado em 15/Set/2011 